\title{
CONCERNING R. L. MOORE'S AXIOM 5*
}

F. B. JONES

In Axiom $5 \dagger$ of his Foundations of Point Set Theory, R. L. Moore postulates the existence of a simple closed curve having certain properties. Professor Moore pointed out to me, while I was still in one of his classes, that in some respects this was undesirable, and that if possible it would be better to state a simple axiom without this feature from which Axiom 5 would follow as a theorem in the presence of the other axioms assumed up to this point in Foundations, namely, Axioms $0-4$. It is the object of this paper to show that this is possible.

Suppose that $S$, the set of all points, is a space satisfying Axioms 0-4 of Moore's Foundations and the following axiom:

Axıom 5'. If $A$ is a point of a region $R$ and $B$ is a point distinct from $A$, there exists in $R$ a compact continuum separating $A$ from $B$.

Theorem 1. If $P$ is a point of a connected domain $D$, then $D-P$ is connected.

Proof. Suppose that $D-P$ is not connected. There exist two distinct components $H$ and $K$ of $D-P$, each having $P$ on its boundary. Let $A$ and $B$ denote points of $H$ and $K$, respectively, and let $A B$ denote an arc from $A$ to $B$ in $S-P$. $\ddagger$ Let $R$ denote a region lying in $D$, containing $P$ but containing no point of $A B$. By Axiom $5^{\prime}, R$ contains a continuum $T$ separating $A$ from $P$. The continuum $T$ must, therefore, contain a point of $H$ and a point of $K$. Since $T$ is a connected subset of $D-P$, this involves a contradiction.

TheORem 2. The space $S$ contains no local cut points.

Theorem 3. If $A$ and $B$ are distinct points, there exists a simple closed curve containing $A$ and $B . \S$

* Presented to the Society, April 9, 1938.

† If $A$ is a point of a region $R$ and $B$ is a point distinct from $A$, there exists, in $R$, a simple closed curve separating $A$ from $B$. Axioms, theorems, and definitions used, but not explicitly stated in this paper, are those of R. L. Moore's Foundations of Point Set Theory, American Mathematical Society Colloquium Publications, vol. 13, New York, 1932. This book will be referred to as Foundations.

$\ddagger$ The existence of $A B$ follows from Theorem 1 of Chapter 2 and Axiom 3 of Foundations.

$\S$ For an argument to prove Theorem 3 using Theorem 2, the reader is referred to certain portions of the argument for Theorem A on page 54 of the author's paper, Concerning certain topologically flat spaces, Transactions of this Society, vol. 42 (1937), pp. 53-93. 
THEOREM 4. If $A$ and $B$ are distinct points, there exists a simple closed curve separating $A$ from $B$ * $^{*}$

TheOREM 5. If $P$ is a point not belonging to the closed and compact point set $M$, there exists a simple closed curve separating $P$ from $M . \dagger$

THEOREM 6. If the points $A$ and $B$ belong to different components of the closed and compact point set $M$, there exists a simple closed curve separating $A$ from $B$ and containing no point of $M$. $\ddagger$

Theorem 7. If the points $A$ and $B$ are separated from each other by the closed and compact point set $M$, then they are separated from each other by a continuum which is a subset of $M$ and which contains no proper subset that separates $A$ from $B . \S$

THEOREM 8. If $M$ is a compact continuum lying in a domain $D$ and $N$ is a compact continuum containing no point of $M$, then there exists in $D$ a compact continuum $L$ which separates $M$ from $N$.

Proof. Let $B$ denote a point of $N$. For each point $A$ of $M$ there exists a region $R$ containing $A$ and lying in $D-D \cdot N$. By Axiom 5' there exists in $R$ a compact continuum $T$ separating $A$ from $B$. Let $Q$ denote the component of $S-T$ containing $A$, and let $G$ denote the collection of all such domains. Since $G$ covers $M$, there exists a finite subcollection $G^{\prime}$ of $G$ covering $M$. Then the boundary $\beta$ of the domain obtained by adding together the elements of $G^{\prime}$ is a compact subset of $D-D \cdot N$ which separates $M$ from $B$. It follows from Theorem 7 that $\beta$ contains a continuum $L$ separating $M$ from $B$ and, consequently, from $N$.

\section{Theorem 9. No arc separates $S$.}

Proof. Suppose, on the contrary, that there exists an arc separating a point $A$ from a point $B$. By Theorem 7 , this arc contains a continuum $M$ separating $A$ from $B$, such that no proper subset of $M$ separates $A$ from $B$. By Axiom 3, $M$ is non-degenerate. Hence $M$ is an $\operatorname{arc} E F$. Let $\omega$ denote an interior point of $E F$. Neither the arc $E \omega$ nor the arc $F \omega$ of $E F$ separates $A$ from $B$. Hence there exist two arcs $A O B$ and $A P B$ from $A$ to $B$ such that (1) $A O B \cdot F \omega=0$, (2) $A P B \cdot E \omega=0$, (3) $A O B \cdot E \omega$ is an arc $T_{o}$, and $O$ is the first point

* Loc. cit., p. 57 for a proof of this theorem.

$\dagger$ Loc. cit., p. 58 for a proof of this theorem.

$\ddagger$ Theorem 6 may be proved by using Theorem 5 and a slight modification of R. L. Moore's argument for Theorem 10 on page 185 of Foundations.

$\S$ For a proof of Theorem 7 see the proof of Theorem 24 on page 194 of Foundations. It is valid without modification for the space considered here. 
of $E \omega$ in $T_{O}$, (4) $A P B \cdot F \omega$ is an arc $T_{P}$, and $P$ is the first point of $F \omega$ in $T_{P}$, and (5) $A O B \cdot A P B$ is two arcs $A A^{\prime}$ and $B B^{\prime}$.* There exists in $A O B+A P B$ a simple closed curve $A^{\prime} O B^{\prime} P A^{\prime}$ whose common part with $E F$ is $T_{O}+T_{P}$. Let $J$ denote this simple closed curve, and let $D$ denote the interior of $J$ with respect to $\omega$ as "the point at infinity." If $D$ contains neither $E$ nor $F$, then it is evident that $E F$ does not separate $A$ from $B$. On the other hand, suppose that $E$ is in $D$. By Theorem 6 , there exists a simple closed curve $C$ separating $\omega$ from $O$ and containing no point of the closed and compact point set $F \omega+E O$ (of $E \omega)+A O B$. It is clear that both $C$ and $J$ are bounded, and that $C$ encloses the point $O$ of $J$ but encloses neither $P$ nor, consequently, the interior of $J . \dagger$ By Theorem 11 on page 163 of Foundations, there exists a simple closed curve $Q$ such that (1) $Q$ is a subset of $C+J$, (2) the interior of $Q$ is a subset both of $D$ and of $I$, the interior of $C$, and (3) $Q$ contains a segment $T$ of $C$ which lies wholly in $D$ but whose extremities separate $O$ from $P$ on $J$. By (1) and (2), $\bar{T}$ contains no point of $E F$ not in $T_{o}$, and by (3), $\bar{T}$ contains no point of $T_{o}$. Hence $T+A P B$ contains an arc from $A$ to $B$ not intersecting $E F$, which is a contradiction.

THEOREM 10. If $A B$ is an arc lying in a connected domain $D$, then $D-A B$ is connected.

Proof. Suppose the contrary. Then there exist two distinct components $H$ and $K$ of $D-A B$, each having boundary points in $A B$. Let $X$ and $Y$ denote points of $H$ and $K$, respectively. Since, by Theorem $9, A B$ does not separate space, there exists in $S-A B$ an arc $X Y$ from $X$ to $Y$. By Theorem $8, D$ contains a continuum $L$ separating $A B$ from $X Y$. Hence $L$ contains both a point of $I I$ and a point of $K$, which is a contradiction.

THEOREM 11. If a connected domain $D$ is regarded as a space in which the term "region" is interpreted to mean a connected open subset of $D$, then with respect to this interpretation of "point" and "region," Axioms 0-4 and 5' are satisfied, and "limit point" is invariant under this change.

Proof. By Theorem 9 on page 96 of Foundations, Axioms 0-2 are satisfied. Theorem 1 of this paper shows that Axiom 3 is satisfied, and it is evident that Axiom $5^{\prime}$ is satisfied. It remains to be shown that Axiom 4 is satisfied.

* Of course, either or both of the $\operatorname{arcs} A A^{\prime}$ and $B B^{\prime}$ may be degenerate. In any case the argument is unaffected.

$\dagger$ This portion of the argument will likely be confusing if the reader does not have in mind the convention and definitions stated on page 153 of Foundations. 
Let $J$ denote a simple closed curve lying in $D$. From Theorem 10 it follows that every component of $D-J$ has $J$ for its complete boundary with respect to $D$. It follows from Axiom 4 that there are at least two such components. Suppose that there are more than two. Let $H$ and $K$ denote two of these components which lie in the same component of $S-J$. Then there exists an arc $T$ not intersecting $J$ which contains a point of $H$ and a point of $K$. By Theorem 8 , there exists in $D$ a continuum separating $J$ from $T$ in $S$. This continuum must, therefore, contain both a point of $H$ and a point of $K$, which is a contradiction.

THEOREM 12. If $A$ is a point of a regior $R$, and $B$ is a point distinct from $A$, there exists in $R$ a simple closed curve separating $A$ from $B$.

Proof. Let $D$ denote a connected domain continuing $A$ and lying in $R$. By Theorem 8 , there exists a continuum $L$ lying in $D$ and separating $A$ from $B$. It follows from Theorems 5 and 11 that there exists in $D$ a simple curve $J$ such that $D-J$ is the sum of two mutually exclusive connected domains $I$ and $E$ both having $J$ on their boundaries, with $I$ containing $A$ and $E$ containing $T$. But $I$ and $E$ must lie in different components of $S-J$ since $I+E=D-J$. Hence $J$ separates $A$ from $T$ and, consequently, from $B$ in $S$.

Theorem 13. A space satisfying Axioms $0-4$ and $5^{\prime}$ satisfies Axioms $0-5$.

That Theorem 13 does not remain true, if Axiom $5^{\prime}$ is weakened by omitting the compactness condition on the separating continuum, may be seen by considering the space consisting of all the points of the number plane with positive ordinates together with the origin. If "limit point" is interpreted in the ordinary sense, it is easy to see that Axiom 5 does not hold at the origin.

Likewise, Theorem 13 does not remain true if Axiom 5' is weakened by replacing the words "a compact continuum" by " a closed and compact point set." An example to show this is included in a paper by the author which is as yet unpublished. *

It may be of interest to the reader that there exists a space satisfying Axioms 0-5 which, even though metric and completely separable, is, nevertheless, not homeomorphic with a subset of a plane or a sphere. $\dagger$

The University of Texas

* Certain equivalences and subsets of a plane. Presented to the Society, June 18, 1936. For the abstract see this Bulletin, vol. 42 (1936), p. 484.

$\dagger$ An example of such a space has been discovered by R. L. Moore and, although unpublished, has been well known among his students for some five or six years. 